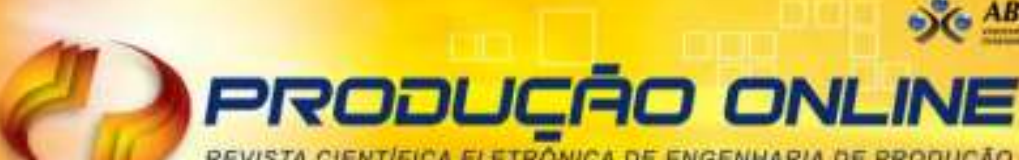 REVISTA CIENTIFICA ELETRÓNICA DE ENGENHARIA DE PRODUCATO

\section{ANÁLISE DA EFICIÊNCIA PRODUTIVA NA INDÚSTRIA AUTOMOTIVA: INTEGRANDO TEORIA DAS RESTRIÇÕES E O ÍNDICE DE RENDIMENTO OPERACIONAL GLOBAL (IROG)}

\section{EVALUATION OF PRODUCTION CAPACITY IN THE AUTOMOTIVE INDUSTRY: NTEGRATING THEORY OF CONSTRAINTS AND THE GLOBAL OPERATIONAL EFFICIENCY INDEX (IROG)}

\author{
Mariane Cásseres de Souza*E-mail: marianecasseres@gmail.com \\ Jéssica Mariella Bauer* E-mail: jehbauer@hotmail.com \\ João William Gauze Junior* E-mail: jwgauze@hotmail.com \\ * Universidade do Vale do Rio dos Sinos (UNISINOS), São Leopoldo, RS
}

\begin{abstract}
Resumo: $O$ objetivo do presente trabalho é analisar a eficiência global de uma célula de manufatura de uma indústria automotiva brasileira. Os objetivos específicos são: (i) analisar o tempo de ciclo por operação em cada produto, (ii) analisar as operações da célula versus demanda. Para tanto se utilizou o modelo de gerenciamento da capacidade proposto por Pacheco et al. (2012), o qual integra os conceitos da Teoria das Restrições e da Total Productive Maintenance (TPM). A aplicação prática do modelo é relevante na medida em que a definição da capacidade produtiva de sistemas possibilita o melhor gerenciamento dos recursos e de suas capacidades, melhora a programação da produção no chão de fábrica e o atendimento às demandas impostas pelo mercado. Os resultados obtidos permitiram comprovar a eficácia do modelo. Foi possível determinar a capacidade do sistema produtivo e o impacto sobre a capacidade produtiva de todo o sistema, ao se considerar as não conformidades de qualidade que ocorrem após o recurso gargalo do fluxo produtivo. O IROG como ferramenta possibilita a tomada de ações mais assertivas na solução de problemas, otimização no uso dos recursos industriais e uma maior integração entre as diversas áreas que englobam a manufatura.
\end{abstract}

Palavras-chave: Capacidade produtiva. Teoria das Restrições. Índice de Rendimento Operacional Global.

Absctract: The objective of the present work is to analyze the global efficiency of a manufacturing cell of a Brazilian automotive industry. The specific objectives are: (i) analyze the cycle time per operation in each product, (ii) analyze the operations of the cell versus demand. For this, the capacity management model proposed by Pacheco et al. (2012), which integrates the concepts of Theory of Constraints and Total Productive Maintenance (TPM). The practical application of the model is relevant as the definition of the productive capacity of systems enables better management of resources and capacities, improve production scheduling on the factory floor and meeting the demands imposed by the market. The results obtained allowed to prove the effectiveness of the model. It was possible to determine the capacity of the productive system and the impact on the productive capacity of the whole system, considering the quality nonconformities that occur after the bottleneck of the productive flow. The IROG as a tool makes it possible to take more assertive actions in problem solving, optimization of the use of industrial resources and greater integration between the different areas that encompass manufacturing.

Keywords: Productive capacity. Theory of Constraints. Indication of Overall Operation Performance. 


\section{INTRODUÇÃO}

Tem-se observado a modernização da indústria brasileira a partir da década de 1990, com a realização de investimentos em novas instalações e equipamentos, e evolução de técnicas e práticas de gestão de produção e operações (ANTUNES et al., 2008). O método de gestão do posto de trabalho tem sido utilizado pelas organizações com o intuito de maximização da utilização dos ativos, aproveitando a capacidade instalada assegurando a sobrevivência e o crescimento das empresas no mercado competitivo atual (ANTUNES et al., 2013). A busca constante pela excelência no âmbito empresarial tem exigido um conhecimento minucioso de todos os recursos da empresa, principalmente no que se refere à área produtiva, na qual se agrega valor ao produto final. O mapeamento dos processos, a eliminação de perdas e o controle por meio de indicadores confiáveis são fundamentais para a melhoria da gestão de recursos e para o apoio à tomada de decisões (PRATES; BANDEIRA 2011).

Determinar a capacidade produtiva de um sistema com maior assertividade é uma das questões essências da Engenharia de Produção, no entanto usualmente as empresas não utilizam métodos científicos e também desconsideram a eficiência real dos equipamentos para determinação da capacidade produtiva (ANTUNES et al., 2008). Contudo, atualmente é de fundamental importância a medição de desempenho dos equipamentos em um sistema de produção o que impacta diretamente na produtividade dos processos, eficiência da mão-de-obra e no nível de qualidade dos produtos (ANDRADE; SCHERER, 2009).

Uma ferramenta para a gestão de postos de trabalho é o Índice de Rendimento Operacional Global (IROG), pelo qual é possível calcular e analisar a eficiência dos recursos disponíveis no processo produtivo (PIRAN et al., 2015) ele é considerado um indicador operacional, aplicável de diversos níveis dentro de um sistema de manufatura (NAKAJIMA, 1988).

A utilização do indicador IROG permite que as empresas analisem as reais condições da utilização de seus equipamentos. Essas análises ocorrem a partir da identificação das perdas existentes nos recursos, envolvendo índices de disponibilidade, desempenho e qualidade (PRATES; BANDEIRA 2011). Conhecendo as restrições do sistema, pode-se maximizar o processo para a empresa atingir sua meta, qual seja, auferir o maior lucro possível ou mesmo identificar a necessidade de 
adquirir ou não novos equipamentos (GASPERIN; PALOMINO, 2006). Além disso, a definição da capacidade produtiva de um sistema possibilita o melhor gerenciamento dos recursos e de suas capacidades, melhora a programação da produção no chão de fábrica, o atendimento às demandas impostas pelo mercado (PACHECHO et al., 2012) e contribui para uma visão mais clara e objetiva dos desperdícios (SILVEIRA JUNIOR; NUNES, 2015).

O IROG permite, a partir de uma medição simplificada, indicar áreas onde devem ser desenvolvidas melhorias, bem como pode ser utilizado como Benchmark, permitindo quantificar as melhorias desenvolvidas nos equipamentos, células ou linhas de produção ao longo do tempo. A análise do IROG de um grupo de máquinas de uma linha ou célula de manufatura permite que se identifique o recurso com menor eficiência, possibilitando, desta forma, a focalização de esforços neste recurso (CHISTÉ, 2012).

Ao verificar a literatura percebe-se que diversos autores tratam os conceitos referentes ao cálculo do IROG com o intuito de evidenciar as diferenças dos cálculos da produtividade efetiva total do equipamento (TEEP) e o índice de eficiência global do equipamento (OEE) (CHISTÉ, 2012; PIRAN et al., 2015; PRATES; BANDEIRA, 2011). Já a abordagem que integra TPM, IROG e a TOC foi mencionada na literatura por alguns autores (JEONG; PHILLIPS, 2001; ROSE et al., 1995; CHAKRAVORTY; ATWATER, 2006; PACHECHO et al., 2012). A TOC - Teorias das Restrições proposta por Goldratt (1984) afirma que através da identificação da restrição do sistema (gargalo), recurso que limita a capacidade da empresa, e elevação da capacidade do mesmo pode-se elevar a capacidade do sistema como um tudo. Já a Total Productive Maintenance (TPM) por meio do IROG apresentado por Nakajima (1989), monitora o rendimento dos equipamentos e dos processos produtivos e tem por objetivo a obtenção de melhores resultados utilizando o aumento do rendimento dos recursos operacionais.

De acordo com Jeong e Phillips (2001), a TPM enfatiza a manutenção autônoma e que os recursos gargalos tenham maior prioridade em relação aos recursos não gargalos dos sistemas produtivos. O IROG, segundo Rose et al., (1995), quando aplicado ao recurso gargalo, pode aumentar o ganho do sistema. Sendo assim, de forma geral pode-se concluir que o gerenciamento de gargalos sugerido pela TOC, somando-se ao indicador IROG oriundo da abordagem da TPM, podem 
contribuir não só para efetivo aumento da produtividade, como também para elevar o ganho do sistema (PACHECO et al., 2012).

Este trabalho busca responder o seguinte problema de pesquisa: "Qual a eficiência de uma célula de manufatura da indústria automotiva"?. O presente artigo tem por objetivo analisar a eficiência global de uma célula de manufatura. Os objetivos específicos são: (i) analisar o tempo de ciclo por operação em cada produto, (ii) analisar as operações da célula versus demanda. Para tal fim, verificou-se a aplicabilidade do modelo de Pacheco et al. (2012) sobre a eficiência da célula o qual permite calcular o índice de rendimento operacional global (IROG) integrando os conceitos da Teoria das Restrições e da Total Productive Maintenance (TPM). A aplicação do método permite identificar a operação gargalo e demais pontos limitantes do processo, contribuindo para a alavancagem da eficiência na empresa. A aplicação do Índice de Rendimento Operacional Global (IROG) é uma ferramenta pelo qual é possível calcular e analisar a eficiência dos recursos disponíveis no processo produtivo. O levantamento de dados foi realizado através de um estudo de caso de natureza descritiva com abordagem qualitativa e quantitativa em uma empresa multinacional da indústria automotiva localizada no sul do Brasil.

Uma breve revisão da literatura sobre capacidade produtiva, TPM, TOC e o IROG servem de pano de fundo para a discussão dos resultados obtidos nesta pesquisa.

\section{REFERENCIAL BIBLIOGRÁFICO}

\subsection{Capacidade Produtiva Industrial}

Existe uma alta complexidade na definição ou medição da capacidade produtiva de um sistema, devido a causas internas e externas, e enfrentada pela variabilidade nas taxas de produção, confiabilidades dos equipamentos, confiabilidades dos fornecedores (HAYES et al., 2008). De acordo com Hopp e Spearman (2001) as variabilidades existentes nos sistemas afetam o desempenho e, com isso, dificultam a tarefa de definir, medir e compreender a real capacidade existente no sistema.

Hopp e Spearman (2001) apresentam os pressupostos para o gerenciamento da capacidade produtiva em sistemas: i) uma linha de fluxo desequilibrada com um 
gargalo conhecido é mais fácil de administrar e demonstra um comportamento logístico melhor quando comparado com uma linha em equilíbrio, onde os tempos de processamento das operações são semelhantes; ii) a capacidade está geralmente disponível apenas em tamanhos incrementais predeterminados, ou seja, podemos comprar um ou dois recursos, mas não um e meio e pode ser impossível ajustar a capacidade de determinada operação com a meta de produção; iii) o custo da capacidade, geralmente, não é o mesmo em cada estação de trabalho e é mais barato manter capacidade em excesso em algumas estações do que em outras.

Segundo Antunes et al. (2008) a capacidade, em unidade de tempo, de um recurso pode ser determinado pela equação 1 e a demanda do recurso pela equação 2.

$$
C=T_{t} \times \mu_{g}
$$

Onde:

C: Capacidade de produção para a produção;

Tt: Tempo total disponível para a produção (tempo);

$\mu g$ : IROG.

$$
D=\sum_{i=1}^{N} t p_{i} \times q_{i}
$$

Onde:

D: Demanda de produtos no equipamento (tempo);

Tpi: Taxa de processamento da peça i no equipamento (tempo por unidade de produção);

qi: Quantidade produzida da peça i no equipamento (unidades de produção).

\subsection{Manutenção Produtiva Total (TPM) e o IROG}

A Manutenção Produtiva Total (TPM) tem como principal objetivo, estimular o envolvimento dos colaboradores a participarem utilizando a experiência como solução para resolver pequenas correções, exemplo: simples aperto de um parafuso, limpeza de filtros ou até mesmo troca de correia entre outros. A TPM tem como essência atacar a causa raiz dos problemas e não apenas mascará-lo, uma máquina que é acompanhada pelo seu operador tem bem menos chance de sofrer pequenas paradas geradas por defeitos simples (TELLES et al., 2014). 
Para Veit et al. (2011) inúmeras são as técnicas que tem como objetivo a redução de desperdícios, sempre buscando melhores resultados operacionais. Entre estas ferramentas destaca-se a Total Productive Maintenance - TPM, que tem como objetivo o desenvolvimento de uma forma de gestão do processo produtivo no sentido de aumentar o rendimento operacional.

A TPM busca a melhoria contínua no sentido de conscientizar o operador da importância do bom desempenho do equipamento para a empresa. De acordo com Telles et al., (2014), os objetivos da TPM são divididos em cinco importantes pontos: a) maximização do IROG do equipamento; b) enfoque sistêmico e globalizado, no que dia respeito ao ciclo de vida do próprio equipamento; c) participação e integração de todos os departamentos envolvidos, como PCP, manutenção e produção; d) envolvimento de todos os níveis hierárquicos da empresa; e) colaboração das atividades de voluntariado desenvolvidas pelos grupos; além da criação de um ambiente propício para o desenvolvimento desses trabalhos.

Através da compreensão da ferramenta IROG, torna-se possível o cálculo e monitoramento constante de eficiência no processo produtivo, elaborando planos de ação visando solucionar os principais motivos de ineficiência produtiva (TELLES et al., 2014).

Conforme Antunes (2008), o índice de multifuncionalidade considera tudo que compõe o sistema: produtos, máquinas e homens em um arranjo produtivo (células, seções, departamentos, minifábricas, etc.)

Para avaliar o indicador de eficiência dos equipamentos, é necessário conhecer como este índice é calculado. Esta eficiência é calculada pelo IROG, proposto por Nakajima (1989).

O IROG pode ser calculado pela multiplicação do Índice do Tempo Operacional (ITO) pelo Índice de Performance Operacional (IPO) pelo Índice de Produtos Aprovados (IPA), conforme a equação 3.

$$
\mu_{\text {global }}=\mu_{1} \times \mu_{2} \times \mu_{3}
$$

O Índice do Tempo Operacional (ITO) consiste no tempo total em que a máquina ficou disponível para produção, excluindo as paradas que ocorreram por 
diversos motivos, ou seja, é o tempo programado para trabalhar que a máquina realmente trabalhou (OLIVEIRA; KLIPPEL, 2004).

$$
\boldsymbol{\mu 1}=I T O=\frac{\text { Tempo de carga }- \text { paradas programadas e não programadas }}{\text { Tempo de carga }}
$$

Quanto menor o valor do ITO, maior a utilização do posto de trabalho, fato este que ocorre porque um baixo valor no índice indica que o equipamento sofreu paradas não programadas. Quando a pouca variedade de peças, o índice é elevado devido a pouca necessidade de trocas de equipamentos, preparações e ajustes.

O Índice de Performance Operacional (IPO) representa os tempos de operações em vazio, paradas momentâneas e quedas de velocidade, ou seja, está relacionado ao desempenho do equipamento (OLIVEIRA; KLIPPEL, 2004).

$$
\boldsymbol{\mu} \mathbf{2}=I P O=\frac{\text { Tempo operacional }-\sum \text { quedas de velocidade }}{\text { Tempo operacional }}
$$

De acordo com Pacheco et al., (2012), quando o valor do IPO é baixo podem ocorrer duas causas fundamentais: i) operações em vazio por falta de alimentação de peças (não havendo assim agregação de valor); ii) não registro dos dados no diário de bordo ou sistema de coleta.

O Índice de Peças Aprovadas (IPA) avalia o índice de rejeição da produção de um lote, equipamento ou setor e que precisam ser retrabalhado (OLIVEIRA; KLIPPEL, 2004).

$$
\boldsymbol{\mu 3}=I P A=\frac{\sum \text { Peças produzidas }-\sum \text { peças rejeitadas } / \text { retrabalhadas }}{\sum \text { peças produzidas }}
$$

Existe outra maneira de calcular o Índice de Eficiência Global, por meio do somatório da multiplicação da quantidade de produtos feitos no equipamento multiplicado pelo tempo de ciclo dos mesmos, dividido pelo tempo disponível deste equipamento, conforme equação7. O conceito do IROG pode ser baseado pelo Índice 
de Eficiência Global dos Equipamentos - OEE (Overall Equipment Effectiveness), quando a capacidade do equipamento analisado não

é considerado crítico, onde o mesmo possui uma capacidade de produção maior que a demanda, ou pela Produtividade Efetiva total do Equipamento - TEEP (Total Effective Equipment Productivity), quando o equipamento é considerado recurso com capacidade restrita (CCR's), ou seja, sua capacidade de produção é igual a sua demanda, pois para atender a demanda neste caso, o equipamento não pode ter paradas, nem as programadas (ANTUNES et al., 2008; VEIT et al., 2011).

$$
\boldsymbol{U}_{\text {global }}=\frac{\sum_{i=1}^{n} \text { tpi } x \text { qi }}{T}
$$

Nakajima (1989) propõe para empresas Classe Mundial no ramo metal mecânico a obtenção de IROG superior a $85 \%$, sendo necessário, para tanto, que o Índice de Disponibilidade seja superior a 90\%; o Índice de Desempenho seja superior a 95\% e o Índice de Qualidade seja superior a 99\%.

De acordo com Hansen (2002) os gestores normalmente supõem que a eficiência global de sua empresa gira em torno de $80 \%$ a $100 \%$, porém ao fazer uso do cálculo constata que os reais números estão muitos a quem destes valores.

Contudo há uma grande necessidade das organizações de identificar a real capacidade de suas máquinas e equipamentos, tratando-se de um grande passo para o aumento de sua competitividade, dando ênfase na redução de todos os tipos de desperdícios (GHINATO, 1996).

\subsection{Teoria das Restrições (TOC) e o IROG}

A Teoria das Restrições, conhecida como TOC (Theory of Constraints) foi proposta pelo doutor em física israelense Eliyahu Goldratt. Segundo esta teoria, uma restrição é qualquer elemento que afete negativamente um sistema, dificultando a obtenção de um melhor desempenho em relação a sua meta, mas que também pode ser explorado de forma que se obtenha uma melhoria de seus resultados (SOARES et al., 2009), ou seja, restrições limitam o ganho da empresa. A proposta da TOC é 
que o gerenciamento eficaz das restrições permita a maximização dos lucros e à melhoria contínua e do ganho.

A metodologia possui foco central nas restrições, ou seja, o elo mais fraco, que pode interferir no desempenho do sistema como um todo e é orientada ao ganho (meta da empresa) e simultaneamente redução do inventário e despesas operacionais (BAUER et al., 2015). Para tanto, a empresa deve buscar otimizar os processos pela melhoria global do sistema com apoio nos cinco passos de focalização ou de melhoria contínua. Deve-se evitar o simples aumento da eficiência local dado que a soma dos ótimos locais não é igual ao ótimo global do sistema (LUCHESE et al., 2016).

Para isso, Goldratt (1996) propõe os cinco passos de focalização da TOC da seguinte maneira:

Passo 1 - Identificar as restrições do sistema;

Passo 2 - Utilizar da melhor maneira possível as restrições do sistema;

Passo 3 - Subordinar todos os demais recursos a decisão tomada no Passo 2;

Passo 4 - Elevar a Capacidade das Restrições;

Passo 5 - Voltar ao passo 1, não deixando que a inércia tome conta do sistema

As medidas da TOC para desempenho são: (i) Ganho (G) corresponde ao preço de venda menos o montante de valores pagos a fornecedores pelos itens relacionados com os produtos vendidos; (ii) Inventário (I): É todo o dinheiro que o sistema investe na compra de coisas que pretende vender, deve ser entendido no seu sentido mais amplo, incluindo máquinas, equipamentos, instalações, construções, materiais, estoques e etc. e (iii) Despesas Operacionais (DO): É todo o dinheiro que o sistema gasta transformando Inventário em Ganho. Ë tudo que temos que colocar na empresa para o seu funcionamento, como salários, aluguéis, luz, encargos, depreciações, etc (CORBETT, 1997; GUERREIRO, 1996).

A TOC apresenta apenas três maneiras de se elevar o lucro, são eles: a) Aumento de ganhos (vendas); b) Redução de investimentos - especialmente em inventários; ou c) Redução de despesas operacionais (custos fixos) (NOREEN et al., 1996).

Com a utilização da ferramenta IROG existem três importantes conceitos: i) gargalos; ii) recursos com capacidade; e, iii) recursos com problema de qualidade. Desta forma as perdas e desperdícios assumem muitas formas e podem ser 
encontradas em qualquer lugar da produção (ANTUNES, 2008; SINGH et al., 2010). Segundo essa teoria, gargalo é um estreitamento na saída de processo produtivo que impede o próprio processo de ser mais eficiente, pois tudo que vem depois, ou mesmo antes deste, fica dependente da restrição imposta pelo gargalo. Um gargalo pode estar antes, no meio ou na saída de um processo ou, até mesmo, ser um fator externo, como o mercado (SOARES et al., 2009). O processo dito como gargalo possui capacidade disponível inferior que a capacidade necessária para atender a demanda do mercado, frente a isso, determina o ritmo em que o processo produtivo será executado (TELLES et al., 2014).

Aumentando a eficiência no gargalo a organização obtém ganhos, através de uma diminuição dos custos de produção, quando, por exemplo, este aumento de eficiência possibilita a redução dos turnos de produção para atendimento da demanda. Outros benefícios favoráveis com o aumento da eficiência do gargalo é o aumento da lucratividade, é o fornecimento de maior flexibilidade para a fábrica, na medida em que é possível aumentar o número de preparações (setups) e, consequentemente, reduzir o tamanho dos lotes (ANTUNES, 2008).

A utilização da ferramenta IROG em um equipamento gargalo aumenta o ganho (aumento da produção) e diminui as despesas operacionais e o inventário (os estoques do gargalo diminuem e a necessidade de pessoal para movimentação do mesmo também cairá) (VEIT et al., 2011), conforme apresentado na Figura 1.

Figura 1 - Avaliação do Impacto do IROG do recurso gargalo nos indicadores Operacionais da TOC

\begin{tabular}{|c|c|}
\hline \multicolumn{2}{|c|}{ IROG NO GARGALO } \\
\hline GANHO & \\
\hline INVENTÁRIO & \\
\hline DESPESAS OPERACIONAIS & \\
\hline
\end{tabular}

Fonte: VEIT et al. (2011)

Os recursos com capacidade possuem a capacidade de produção superior à demanda de mercado, porém, quando estes recursos não são devidamente programados e gerenciados, podem apresentar os efeitos de um recurso de gargalo 
através de um pico de desbalanceamento entre a sua demanda e respectiva capacidade de falta de matéria-prima, mix de produtos, problemas associados a manutenção, preparação de máquina, podendo gerar outro problema de custos e o nível elevado de estoques (MONDEN, 1983; OHNO, 1988; CONBOY, 2009). Quanto aos recursos com problema de qualidade, são também ditos como críticos, visto que a gestão se faz importante, principalmente quando estes recursos se localizarem após um recurso gargalo no fluxo de produção (ANTUNES; KLIPPEL, 2012).

\subsubsection{O algoritmo Tambor-Pulmão-Corda (TPC)}

O TPC baseia-se é estruturado nos mecanismos: Tambor $(T)$, Pulmão $(P)$ e Corda (C). (LUCHESE et al., 2016). A ideia central do algoritmo TPC consiste em estabelecer a produção puxada para controlar o fluxo de inventário no sistema produtivo (WATSON; PATTY, 2009). E, portanto, os recursos com menor capacidade que os gargalos devem ser considerados o tambor (T) da fábrica (GOLDRATT; FOX, 1989). O TPC possibilita a melhoria da tomada de decisão e planejamento das ações em caso de atraso na produção por quebra de máquina ou defeitos, e o gargalo não perde tempo de produção por qualquer parada imprevista (LUCHESE et al., 2016).

Por intermédio do método TPC da teoria das restrições é possível localizar o elemento que restringe o sistema, denominado de gargalo ou recurso com restrição de capacidade (RRC), e estabelecer um ritmo de produção para todo o sistema (SOUZA, 2006).

Após a identificação do tambor da fábrica, todos os recursos que antecedem o mesmo serão puxados com ritmo igual ou pouco superior ao ritmo da restrição, representando assim, a programação para trás. Os recursos após os gargalos serão empurrados até o final do sistema produtivo, representando assim, programação para frente (GOLDRATT; FOX, 1989; SOARES et al., 2009).

Os recursos restritivos, representados pelos tambores, recebem atenção especial na fábrica. Muitas vezes, o desempenho econômico financeiro da indústria é representado pelos recursos críticos. Assim sendo, o Tambor deve ser protegido quanto aos eventuais problemas que possam ocorrer nas máquinas que o antecedem. Esses problemas podem ser exemplificados como variabilidades de tempos de 
processo, problemas associados à qualidade, quebra de máquinas, falta de matériasprimas, entre outros (GOLDRATT; FOX, 1989).

O pulmão $(P)$ é a segurança colocada antes do tambor para evitar paradas devido ao impacto das variabilidades, como exemplo: quebra de máquinas, variação no tempo de processo, problemas de qualidade, falta de matéria prima para produzir, etc. Há três tipos de pulmão que podem ser usados nesse caso: pulmão de tempo, inventário ou de capacidade (PACHECO et al., 2012; SOARES et al., 2009).

A corda $(C)$ no algoritmo tem como objetivo sinalizar a necessidade de entrada de materiais para a alimentação do gargalo e dos pulmões que antecedem as linhas de montagem. Portanto, através da sinalização ocorrerá a liberação de materiais somente na quantidade e na hora certa, sua função é de limitar o índice pela qual a matéria-prima é liberada para a fábrica (GOLDRATT; FOX, 1989). Ela assegura que a restrição não receba excesso ou falta de ordens, e sincroniza novas liberações no início da linha com o conteúdo do pulmão (SRINIVASAN et al., 2004).

\section{METODOLOGIA}

O estudo caracteriza-se como uma pesquisa qualitativa de caráter exploratório, sob a forma de um estudo de caso. O estudo de caso trata-se de uma investigação empírica, que investiga um fenômeno contemporâneo inserido em um contexto, especialmente quando os limites entre o fenômeno e o contexto não estão claramente definidos (YIN, 2005).

Quanto à técnica de coleta de dados utilizou-se a observação participante, documentos, planilhas e relatórios, sobre o aspecto qualitativo, que demonstram a utilização de ferramentas que auxiliam no acompanhamento de melhoria contínua dos processos e aprimoramento do IROG.

Neste sentido, o objetivo do estudo consiste na aplicação da ferramenta IROG, evidenciando princípios através da TOC e aumento da eficiência no posto de trabalho, de uma empresa do ramo automotivo. A Figura 2 apresenta as etapas utilizadas para o desenvolvimento deste estudo. 
Figura 2 - Etapas do estudo

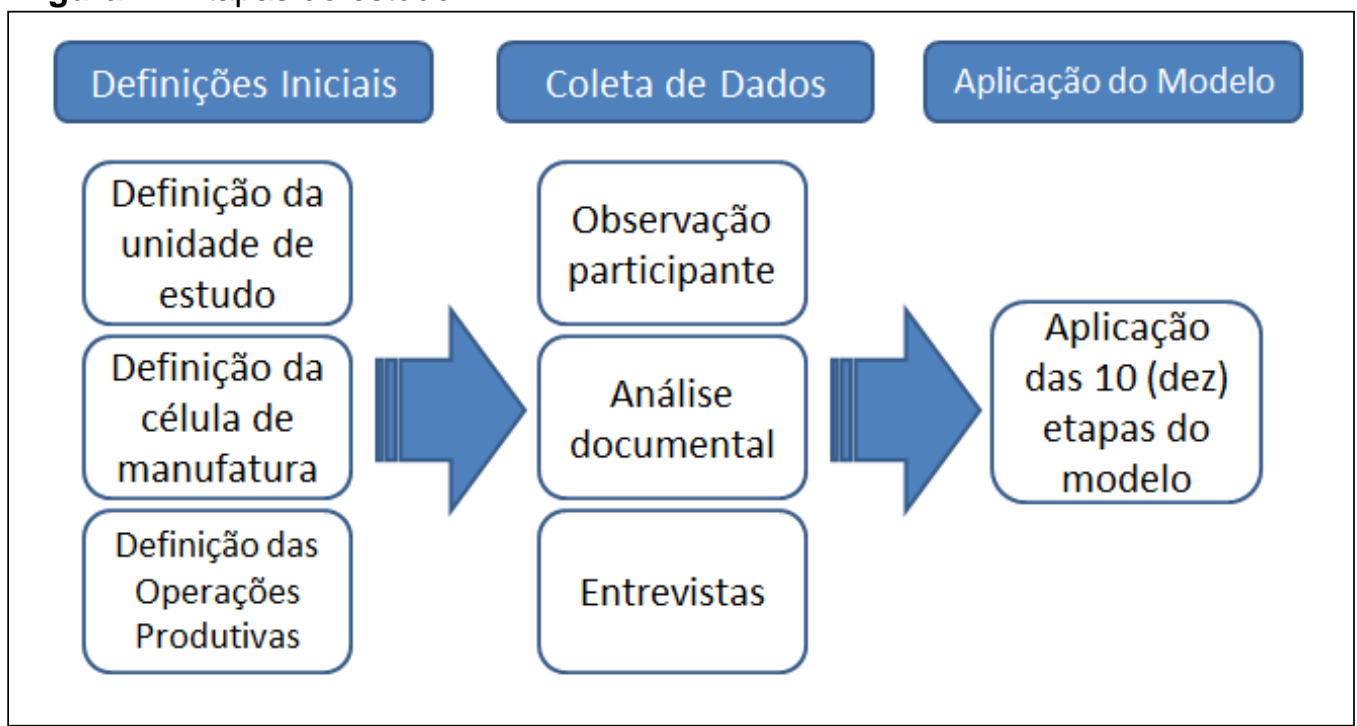

Fonte: Elaborado pelos autores (2017)

Inicialmente definiu-se a unidade de estudo que foi composta por uma empresa multinacional do ramo automotivo localizada no estado do Rio Grande do Sul, Brasil.

A empresa atende a todas as grandes montadoras do país com uma expressiva parcela de participação no mercado nacional. Hoje, cerca de $85 \%$ dos veículos produzidos no país são abastecidos por componentes fornecidos pela empresa em estudo. Foram efetuados acompanhamentos em uma célula de manufatura dentro de uma das unidades que compõem a empresa, durante uma semana.

Analisaram-se as operações produtivas referentes à usinagem, conformação e tratamento térmico, operações na qual ocorria transformação do produto, não foram consideradas etapas de inspeções e transportes.

Foram observados os ciclos de produção, considerando o início e fim de ciclo conforme orientações nas folhas de operação. Além das folhas de operações que informam as etapas do processo, tempos de ciclo e especificações do produto em cada uma das etapas, foram consultados também os programas de produção que informam a demanda mensal de cada produto, as marcações de produção horária em relação à produção prevista e os cartões de sucata que informam o nome do produto e número de operação correspondente.

Foram entrevistados os operadores de máquina, operadores multifuncionais e o coordenador de produção. Todos foram questionados em relação aos tempos de ciclo, índice de não qualidade e sobre a identificação do gargalo. 


\subsection{Modelo de Pacheco et al. (2012)}

Na sequencia aplicou-se o modelo desenvolvido por Pacheco et al. (2012) que contempla alguns pressupostos. O primeiro pressuposto assumido para 0 desenvolvimento do método é considerar a restrição da empresa interna, ou seja, podendo ser recurso físico, equipamento, linha de produção, célula, etc. O segundo pressuposto, é em relação à variedade de produtos fabricados independente do volume o mesmo não impacta nos resultados apresentados. E, portanto, o método pode ser replicado em ambientes de manufatura de alto ou baixo nível de customização dos produtos fabricados.

O período de análise não influencia o modelo, portanto pode-se considerar um dia, um mês ou mesmo anos. Outro aspecto fundamental é a confiabilidade das informações de entrada como: quantidades demandadas e principalmente os tempos de processamento. Esse aspecto é um pré-requisito para se definir com precisão qual recurso é a restrição do sistema. O modelo proposto é composto pelas seguintes etapas, conforme se pode observar na Figura 3.

Figura 3 - Etapas do modelo de gerenciamento da capacidade integrando TOC e TPM

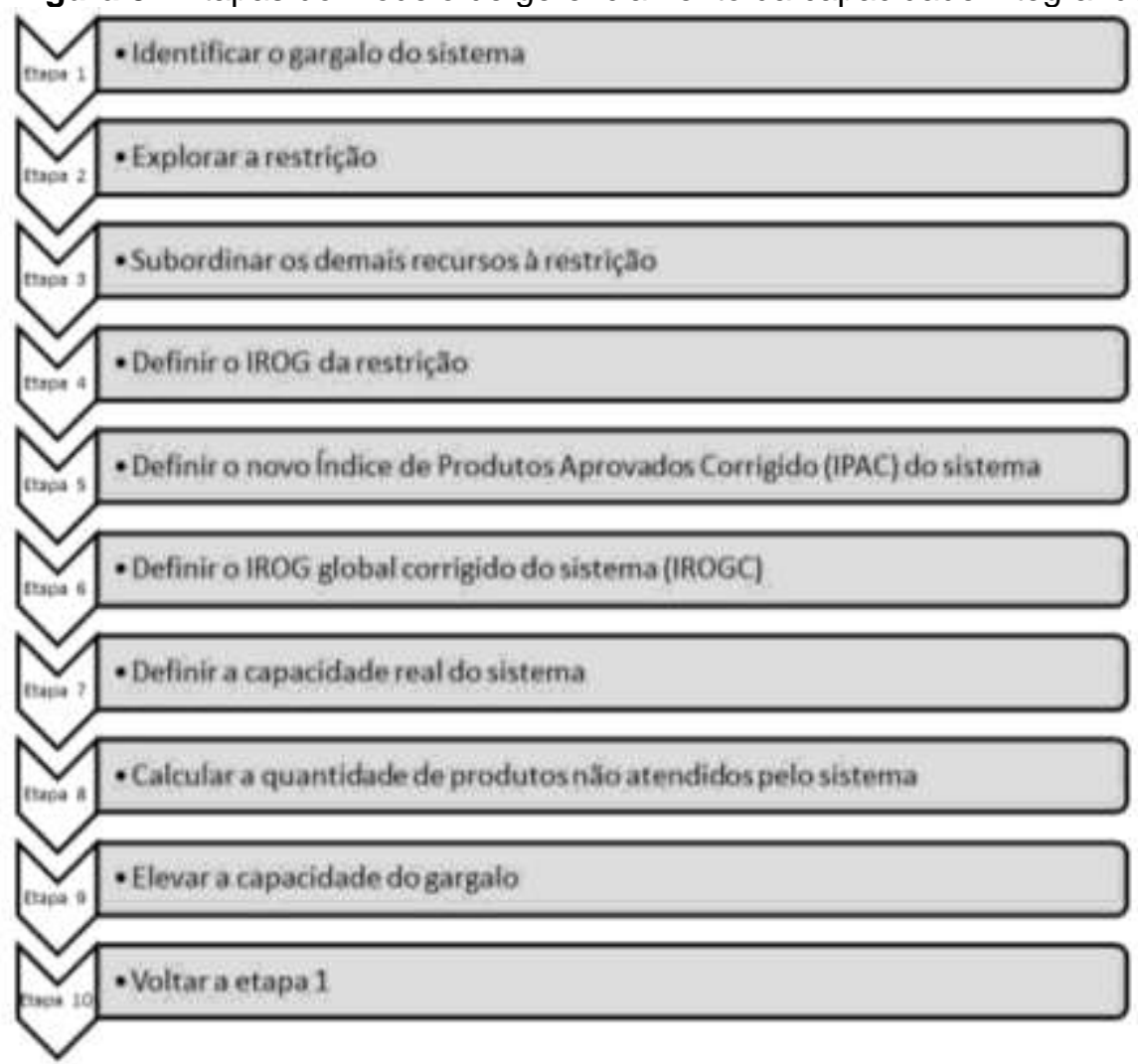

Fonte: Pacheco et al. (2012) 
(1) lidentificar a restrição do sistema, ou seja, o recurso cuja capacidade é inferior à demanda imposta;

(2) Explorar a restrição visando maximizar o ganho do sistema, conforme preconiza o passo 2 do processo de focalização da TOC;

(3) Subordinar os demais recursos à restrição: nessa etapa os recursos que estão dispostos no leiaute do sistema produtivo, tanto à jusante quanto à montante do gargalo, são subordinados ao gargalo, buscando assegurar que a capacidade do gargalo irá definir o planejamento da produção no sistema produtivo;

(4) Definir o IROG (Índice de Rendimento Operacional Global) da restrição: nessa etapa do modelo calcula-se o TEEP do gargalo onde busca-se avaliar o nível atual de desempenho do recurso gargalo do sistema.

(5) Definir o novo Índice de Produtos Aprovados Corrigido (IPAC) do sistema: nessa etapa, calcula-se o IPA de todos os recursos dispostos no fluxo de produção após o gargalo e efetua-se a multiplicação entre esses índices calculados, obtendose assim num valor único, denominado de IPA Corrigido (IPAC). Ao mensurar a quantidade de produtos rejeitados no fluxo após o gargalo, é possível medir o real índice de produtos conformes que são produzidos após o gargalo contribuindo para um dimensionamento mais acurado da capacidade global do sistema.

(6) Definir o IROG Corrigido do sistema: nessa etapa do modelo é obtido o novo IROG do sistema, a partir da visão de capacidade dos gargalos da TOC, denominado nesse estudo de IROGC. O resultado é obtido multiplicando-se o IROG pelo indicador IPAC;

(7) Definir a capacidade real do sistema: nessa etapa do modelo define-se a capacidade real do sistema em atender à demanda a partir da capacidade do gargalo. O resultado é obtido multiplicando-se o tempo disponível para a produção no recurso gargalo pelo valor do IROGC;

(8) Calcular a quantidade de produtos não atendidos pelo sistema: essa etapa do modelo consiste em dividir a diferença temporal do gargalo pelo somatório dos tempos de processamento do produto no recurso gargalo;

(9) Elevar a capacidade do gargalo: nessa etapa são realizadas ações, caso sejam necessárias, para elevar a capacidade do gargalo. como: aplicar as técnicas enxutas de redução de perdas, reduzindo a variabilidade, reduzindo os tempos de setup, adquirindo outros recursos, usando horas extras, contratação de novos 
trabalhadores, usar roteiros alternativos reduzindo o tempo de operação dos produtos no gargalo, e assim por diante.

\section{ESTUDO DE CASO}

A etapa 1 do modelo consiste na primeira etapa dos cinco passos de focalização da TOC. Para identificar o gargalo primeiro usou-se a relação entre capacidade e demanda proposta por Antunes et al. (2008), apresentada na Tabela 1, durante o período de uma semana de produção da célula de manufatura em estudo. Assim multiplica-se a demanda semanal pelos tempos de processamento de cada equipamento obtendo a demanda semanal em unidade de tempo para cada equipamento. A demanda semanal total é a soma das demandas de todos os produtos em cada equipamento. O IROG de cada equipamento foi obtido do sistema de gestão da empresa compreendendo a semana realizada no estudo, após foi multiplicado pelo tempo disponível em cada equipamento para poder conhecer a real capacidade em unidade de tempo de cada equipamento. Por fim, foi possível identificar a restrição do sistema com base na análise da capacidade de cada recurso onde se verificou que o recurso $D$ não poderá atender à demanda.

As etapas 2 e 3 do modelo correspondem às respectivas etapas dos 5 passos de focalização da TOC. O algoritmo TPC da TOC auxilia na aplicação destas duas etapas. Assim colocou-se o pulmão de proteção antes do equipamento (recurso) 4 que é o gargalo do sistema (Tambor). A corda sinaliza a necessidade de entrada de materiais no fluxo, para abastecer o sistema com materiais, limitando a quantidade de matéria-prima conforme a capacidade do gargalo, ilustrado na Figura 4. O IROG do gargalo já foi identificado anteriormente para realizar a etapa 4. Verifica-se nesse caso que o IROG da operação gargalo é de 0,39. Apesar dos recursos $C, E, F$ e $G$ possuírem IROG menor que na operação 4 nenhuma das quatro é gargalo em função da relação capacidade versus demanda. 
Figura 4 - Etapas 2 e 3 do processo de focalização

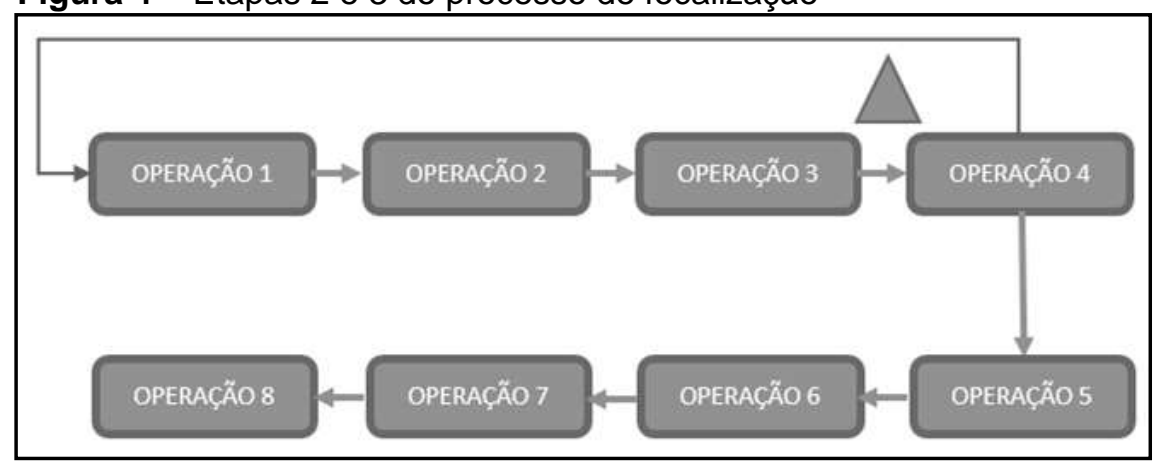

Fonte: Elaborado pelos autores (2017)

Para a etapa 5 é realizado o cálculo do IPAC multiplicando os valores dos coeficientes de qualidade (IPA) individuais de todos os recursos após o gargalo (nesse caso os recursos E, F, G e H). Assim, resultando em: 0,97 x 0,94 x 0,98 x 0,97 = 0,87. O valor 0,87 encontrado significa que a taxa de produtos conformes produzidos após o gargalo é de $87 \%$.

Na etapa 6 calcula-se o IROG corrigido, multiplicando o IROG do gargalo (0.39) da etapa 4 pelo valor do IPAC (0.87) da etapa 5 . Na etapa 7 calcula-se a nova capacidade do gargalo, constatando que antes era de 176904 segundos, e agora é 153333 segundos, reduzindo em 23571 segundos.

Na etapa 8 faz-se o cálculo dos produtos não atendidos pelo sistema produtivo, dividindo a nova diferença de tempo (-26176 segundos) pela média dos tempos de processamento no recurso gargalo 36,87 segundos, resultando em 710 produtos. Então, anteriormente a quantidade de produtos não atendidos pelo sistema era de 95, assim sendo uma diferença de $87 \%$. Na tabela 2 estão expostos os resultados descritos. 
Tabela 1 - Análise capacidade versus demanda

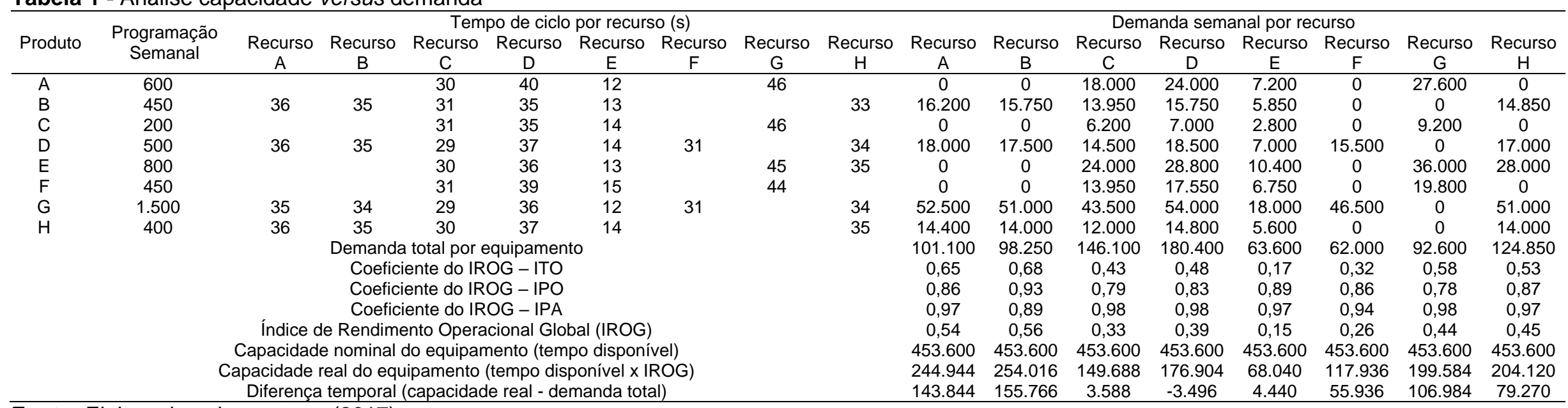

Fonte: Elaborado pelos autores (2017) 
Tabela 2 - Nova relação capacidade versus demanda

\begin{tabular}{|c|c|c|c|c|c|c|c|c|c|c|c|c|c|c|c|c|c|}
\hline \multirow[b]{2}{*}{ Produto } & \multirow[b]{2}{*}{$\begin{array}{l}\text { Programaçã } \\
\text { o Semanal }\end{array}$} & \multicolumn{8}{|c|}{ Tempo de ciclo por recurso (s) } & \multicolumn{8}{|c|}{ Demanda semanal por recurso } \\
\hline & & $\begin{array}{l}\text { Recurso } \\
\text { A }\end{array}$ & $\begin{array}{l}\text { Recurso } \\
\text { B }\end{array}$ & $\begin{array}{l}\text { Recurso } \\
\text { C }\end{array}$ & $\begin{array}{l}\text { Recurso } \\
D\end{array}$ & Recurso & $\begin{array}{l}\text { Recurso } \\
\mathrm{F}\end{array}$ & $\begin{array}{l}\text { Recurso } \\
\text { G }\end{array}$ & $\begin{array}{c}\text { Recurso } \\
\mathrm{H}\end{array}$ & $\begin{array}{l}\text { Recurso } \\
\text { A }\end{array}$ & $\begin{array}{l}\text { Recurso } \\
\text { B }\end{array}$ & $\begin{array}{l}\text { Recurso } \\
\text { C }\end{array}$ & $\begin{array}{l}\text { Recurso } \\
\text { D }\end{array}$ & $\underset{E}{\text { Recurso }}$ & $\begin{array}{l}\text { Recurso } \\
\text { F }\end{array}$ & $\begin{array}{l}\text { Recurso } \\
\text { G }\end{array}$ & $\begin{array}{c}\text { Recurso } \\
\mathrm{H}\end{array}$ \\
\hline A & 600 & & & 30 & 40 & 12 & & 46 & & 0 & 0 & 18.000 & 24.000 & 7.200 & 0 & 27.600 & 0 \\
\hline B & 450 & 36 & 35 & 31 & 35 & 13 & & & 33 & 16.200 & 15.750 & 13.950 & 15.750 & 5.850 & 0 & 0 & 14.850 \\
\hline C & 200 & & & 31 & 35 & 14 & & 46 & & 0 & 0 & 6.200 & 7.000 & 2.800 & 0 & 9.200 & 0 \\
\hline D & 500 & 36 & 35 & 29 & 37 & 14 & 31 & & 34 & 18.000 & 17.500 & 14.500 & 18.500 & 7.000 & 15.500 & 0 & 17.000 \\
\hline $\mathrm{E}$ & 800 & & & 30 & 36 & 13 & & 45 & 35 & 0 & 0 & 24.000 & 28.800 & 10.400 & 0 & 36.000 & 28.000 \\
\hline $\mathrm{F}$ & 450 & & & 31 & 39 & 15 & & 44 & & 0 & 0 & 13.950 & 17.550 & 6.750 & 0 & 19.800 & 0 \\
\hline G & 1.500 & 35 & 34 & 29 & 36 & 12 & 31 & & 34 & 52.500 & 51.000 & 43.500 & 54.000 & 18.000 & 46.500 & 0 & 51.000 \\
\hline \multirow[t]{11}{*}{$\mathrm{H}$} & & 36 & 35 & 30 & 37 & 14 & & & 35 & 14.400 & 14.000 & 12.000 & 14.800 & 5.600 & 0 & 0 & 14.000 \\
\hline & \multicolumn{9}{|c|}{ Demanda total por equipamento } & 101.100 & 98.250 & 146.100 & 180.400 & 63.600 & 62.000 & 92.600 & 124.850 \\
\hline & & 0,65 & 0,68 & 0,43 & 0,48 & 0,17 & 0,32 & 0,58 & 0,53 \\
\hline & \multirow{2}{*}{\multicolumn{9}{|c|}{$\begin{array}{l}\text { Coeficiente do IROG - IPO } \\
\text { Coeficiente do IROG - IPA }\end{array}$}} & 0,86 & 0,93 & 0,79 & 0,83 & 0,89 & 0,86 & 0,78 & 0,87 \\
\hline & \multirow{2}{*}{\multicolumn{9}{|c|}{$\begin{array}{c}\text { Coeficiente do IROG - IPA } \\
\text { Índice de Rendimento Operacional Global (IROG) }\end{array}$}} & 0,97 & 0,89 & 0,98 & 0,98 & 0,97 & 0,94 & 0,98 & 0,97 \\
\hline & & & & & & & & & & 0,54 & 0,56 & 0,33 & 0,39 & 0,15 & 0,26 & 0,44 & 0,45 \\
\hline & \multirow{2}{*}{\multicolumn{9}{|c|}{ Novo coeficiente do IROG - IPAC }} & 0,97 & 0,89 & 0,98 & 0,87 & 0,87 & 0,87 & 0,87 & 0,87 \\
\hline & \multirow{2}{*}{\multicolumn{9}{|c|}{$\begin{array}{l}\text { Novo IROG corrigido do sistema } \\
\text { Capacidade nominal }\end{array}$}} & 0,54 & 0,56 & 0,33 & 0,34 & 0,34 & 0,34 & 0,34 & 0,34 \\
\hline & & & & & & & & & & 453.600 & 453.600 & 453.600 & 453.600 & 453.600 & 453.600 & 453.600 & 453.600 \\
\hline & \multicolumn{9}{|c|}{ Capacidade real do equipamento (tempo disponível x IROG) } & 244.944 & 254.016 & 149.688 & 154.224 & 68.040 & 117.936 & 199.584 & 204.120 \\
\hline & \multicolumn{9}{|c|}{$\begin{array}{l}\text { Diferença temporal (capacidade real - demanda total) } \\
\text { Quantidade de produtos não atendidos }\end{array}$} & 143.844 & 155.766 & 3.588 & -26.176 & 4.440 & 55.936 & 106.984 & 79.270 \\
\hline
\end{tabular}

Fonte: Elaborado pelos autores (2017) 
A partir da análise da aplicação prática do modelo proposto por Pacheco et al.(2012) verificou-se que calculando o IROG corrigido do sistema, o número de produtos não entregues teoricamente aumentou em relação ao cenário inicial que desconsiderava a não qualidade após o gargalo. Esse resultado mostra que este modelo aborda o sistema analisado por meio de uma visão sistêmica, confirmando que a quantidade de produtos rejeitados no sistema após o gargalo impacta diretamente na real capacidade.

\section{CONSIDERAÇÕES FINAIS}

Este artigo teve por objetivo analisar a eficiência global de uma célula de manufatura. Os objetivos específicos são: (i) analisar o tempo de ciclo por operação em cada produto, (ii) analisar as operações da célula versus demanda. Para tal fim, verificou-se a aplicabilidade do modelo de Pacheco et al. (2012) sobre a eficiência da célula o qual permite calcular o índice de rendimento operacional global (IROG) integrando os conceitos da Teoria das Restrições e da Total Productive Maintenance (TPM).

Um estudo de caso foi aplicado em uma unidade de manufatura da indústria automotiva. Dados foram coletados por meio de entrevistas, análise documental e observação participante na empresa em que se desenvolveu o estudo.

O IROG foi adotado como indicador de eficiência e como forma de gestão e melhoria dos equipamentos e processos produtivos com o intuito de focalizar as ações da gestão nos pontos críticos do sistema, que são os equipamentos gargalos e os equipamentos com capacidade restritiva, os CCRs.

Como resultados observou-se que o IROG como ferramenta, se analisado de forma sistêmica possibilita a tomada de ações mais assertivas na solução de problemas, otimização no uso dos recursos industriais e uma maior integração entre as diversas áreas que englobam a manufatura. O IROG é de fundamental importância para quantificar o impacto e, quais equipamentos contribuem para frear o resultado da empresa. Deve-se administrar a capacidade do sistema, considerando seus limites na decisão a ser tomada. Também é necessário ter em mente que o que limita a capacidade de um sistema são suas restrições. 
Como conclusões deste estudo verificou-se que o número de produtos não entregues teoricamente aumentou em relação ao cenário inicial que desconsiderava a não qualidade após o gargalo. Esse resultado mostra que o modelo proposto por Pachecho et al. (2012) aborda o sistema analisado por meio de uma visão sistêmica, confirmando que a quantidade de produtos rejeitados no sistema após o gargalo impacta diretamente na real capacidade.

A pesquisa confirmou a aplicabilidade do modelo de gerenciamento em um estudo de caso real, como sugerem os autores, em uma manufatura com uma variedade grande de peças e mostrou-se eficiente quanto aos indicadores de capacidade propostos, assim como, as etapas definidas.

O estudo de caso auxiliou o processo de tomada de decisão sobre os níveis de capacidade, como também possibilitou o melhor gerenciamento dos recursos e suas capacidades, da programação da produção no chão de fábrica e do atendimento às demandas programadas. Em termos de resultados obtidos pela empresa pode-se destacar: (i) avaliação da capacidade real de produção da linha; ii) possibilidade de focar os esforços para maximizar os ganhos sistêmicos da empresa; e (iii) melhoria em termos de plano de investimento em recursos produtivos.

Os resultados obtidos no estudo comprovaram que a eficácia do modelo e de fácil aplicação prática, assim como foi possível valer-se da unificação das duas teorias e verificar que a real capacidade da célula de manufatura era diferente do que se havia calculado até o momento.

Contudo, como limitação aponta-se a generalização desse estudo de caso em termos populacionais. Por tratar-se de um estudo de caso único, apropriado para o tipo de investigação executada em que se procurava certa profundidade, as possíveis generalizações tornam-se comprometidas. Contudo, as conclusões obtidas a partir desta pesquisa são válidas. Futuras pesquisas são indicadas como a realização de levantamentos quantitativos mais amplos que permitam um maior grau de generalização dos resultados. Outra possibilidade seria a replicação do estudo em outras organizações de outros setores de atuação a partir da metodologia proposta por Pacheco et al. (2012).

Como extensão natural dessa pesquisa, os autores sugerem a aplicação de estudos de casos que utilizem a abordagem de Pacheco et al. (2012) em empresas de diferentes segmentos de atuação, a fim de avaliar a abrangência de aplicação do 
modelo proposto. Além disso, aponta-se para novos horizontes pesquisas que procurem analisar criticamente os conceitos postos de trabalho e novas abordagens. Outra informação relevante para a replicação do modelo, é sua aplicabilidade tanto para sistemas de manufatura de produção puxada como empurrada.

\section{REFERÊNCIAS}

ANDRADE, J.J.O.; SCHERER C.S. Estudo de Caso da Aplicação do Indicador de Eficiência Global de Equipamentos (OEE) para Diagnóstico e Melhoria de Produtividade em uma Linha de Produção Automotiva. ENEGEP - ENCONTRO NACIONAL DE ENGENHARIA DE PRODUÇÃO, 29., 2009. Anais... Salvador, BA, 2009.

ANTUNES JR, A. V. ; ALVAREZ, R. ; KLIPPEL, M.; BORTOLOTTO, P.; PELLEGRIN, I. Sistemas de produção: sistemas e práticas para projeto e gestão da produção enxuta. Porto Alegre: Bookman, 2008.

ANTUNES JR, A. V.; KLIPPEL, M. Uma abordagem metodológica para o gerenciamento das restrições dos sitemas produtivos: a gestão sistêmica unificada integrada voltada aos resultados do posto de trabalho, 2012.

ANTUNES, J. et al. Uma revolução na produtividade: a gestão lucrativa dos postos de trabalho. Porto Alegre: Bookman, 2013.

BAUER, J.M.; VARGAS, A.; SELLITTO, M.A. Comparação entre a Teoria das Restrições, a Manufatura Enxuta e a Abordagem Seis Sigma. Revista Linkania Master, v. 5, n. 1, p.170196, 2015.

CHAKRAVORTY, S. S.; BRIAN ATWATER, J. Bottleneck management: theory and practice. Production, Planning \& Control, v. 17, n. 5, p. 441-447, 2006.

CHISTÉ, M. A Influência do IROG na Gestão e Melhoria Contínua dos Equipamentos e Processos: Estudo de Caso em uma Célula de Manufatura na Empresa Master Sistemas Automotivos Ltda. Revista Global Manager Acadêmica, v. 1, n. 1, 2012.

CONBOY, K. Agility from first principles: reconstructing the concept of agility in information systems development, Information Systems Research, v.20, n.3, p.329-354, 2009.

CORBETT NETO, T. Contabilidade de ganhos: a nova contabilidade gerencial de acordo com a Teoria das restrições. São Paulo: Nobel, 1997.

COX, J; SPENCER, M. Manual da teoria das restrições. Porto Alegre: Bookman, 2002.

GHINATO, P. Sistema Toyota de produção: mais do que simplesmente just-in-time. Caxias do Sul: Editora da Universidade de Caxias do Sul, 1996.

GOLDRATT, E M. \& COX, J.F. A meta. 1. ed. São Paulo SP: Nobel, 1984.

GOLDRATT, E. M. A síndrome do palheiro: garimpando informações num oceano de dados. São Paulo: Editora Educator, 1996. 
GOLDRATT, E. M.; FOX, R. E. A Corrida pela vantagem competitiva. São Paulo: Editora Educador, 1989.

GUERREIRO, Reinaldo. Os princípios da teoria das restrições sob a ótica da mensuraçào econômica. Caderno de Estudos, n. 13, p. 01-10, 1996.

HANSEN, R. C. Overall equipment effectiveness: a powerful production/maintenance tool for increased profits. New York: Industrial Press, 2002.

HAYES, R. P. G.; UPTON, D.; WHELLWRIGHT, S. Produção, estratégia e tecnologia: em busca da vantagem competitiva. Porto Alegre: Bookman, 2008.

HOPP, W.; M. L. SPEARMAN. Factory physics. Boston: Irwin, 2001.

JEONG, K. Y.; PHILLIPS, D.T. Operational efficiency and effectiveness measurement. International Journal of Operation \& Production Management, v. 21, p. 1404-1416, 2001.

LIKER, J.,; MEIER, D. Modelo Toyota - manual de aplicação: um guia prático para a implementação dos 4Ps da Toyota, 2007. Porto Alegre: Bookman.

LUCHESE, J.; BAUER, J.M.; VARGAS, A.; SAUERESSIG, G.; DE SOUZA, M.. SELLITTO, M.A. Implantação da Lógica Tambor-Pulmão-Corda em uma Empresa da Indústria Moveleira. Holos, v. 8, n.31, p.262-276, 2016.

MONDEN, Y. The Toyota production system, Productivity Press, Portland, OR. 1983.

NAKAJIMA, S. TPM development program: implementing total productive maintenance. Productivity Press, Cambridge. Massachusetts, 1989.

NOREEN, E.; SMITH, D.; MACKEY, J. T. A Teoria das Restrições e suas Implicações na Contabilidade Gerencial: um relatório independente. São Paulo: Educator, 1996.

OHNO, T. O sistema Toyota de produção: além da produção em larga escala. Porto Alegre: Bookman, 1997.

OHNO, T. The Toyota production system: Beyond Large-Scale Production, Productivity Press, Portland, OR.1988.

OLIVEIRA, J. C. A; KLIPPEL, A. F. Aumento da eficiência operacional através da abordagem de Gestão dos Postos de Trabalho (GPT): um estudo de caso na indústria de medicamentos. In: ENEGEP - ENCONTRO NACIONAL DE ENGENHARIA DE PRODUÇÃO, 24., 2004. Anais... Florianópolis, SC, 2004.

PACHECO, D. A. J; ANTUNES JR., J.A.V; LACERDA, D. P; GOLDMEYER, D. B; GILSA, C. V; Modelo de Gerenciamento da Capacidade Produtiva: Integrando Teoria das Restrições e o Índice de Rendimento Operacional Global (IROG). Revista Produção Online, v.12, n. 3, p. 806-826, 2012.

PRATES, C.C.; BANDEIRA, D. Aumento de eficiência por meio do mapeamento do fluxo de produção e aplicação do Índice de Rendimento Operacional Global no processo produtivo de uma empresa de componentes eletrônicos. Revista Gestão \& Produção, v. 18, n. 4, p. 705-718, 2011. 
ROSE, E.; ODOM, R.; DUMBAR, R.; HINCHMAN, J. How TOC \& TPM work together to build the quality toolbox of SDWTs. In: Electronics manufacturing technology symposium, manufacturing technologies - present and future, Seventeenth IEEE/CPMT International, Austin, TX , USA, 56-59, 1995.

SILVEIRA JUNIOR, R.J.A.; NUNES, F.L. O Índice de Rendimento Operacional Global e a Troca Rápida de Ferramentas Aliadas à Eficiência de uma Aplicadora de Adesivos. ENEGEP- ENCONTRO NACIONAL DE ENGENHARIA DE PRODUÇÃO, 35., 2015. Anais... Fortaleza, CE, 2015.

SINGH, B., GARG, S. K., SHARMA, S. K., GREWAL, C. Lean implementation and its benefits to production industry, International Journal of Lean Six Sigma, v.1, n.2, p.157168. 2010.

SOARES, I.H.L.; ARMOND-DE-MELO, D.R.; LEOPOLDINO, C.B.; ABREU, J.C.A.; MOITA, F.M. A Teoria das Restrições em um Processo de Fabricação da Indústria na Construção Civil: Um Estudo de Caso. Revista de Administração da UFSM, v. 2, n. 3, p. 463-478, 2009.

SOUZA, A.A.C. Aplicação da metodologia Tambor - Pulmão - Corda (TPC) com supermercado na gestão de manufatura de eletrodos de grafite das unidades de Candeias e Monterrey da Graftech International Ltd.112 f.2006. Dissertação (Mestrado em Administração), Escola de Administração, Universidade Federal da Bahia, UFBA, Bahia, BA, 2006.

SRINIVASAN, M. M.; JONES, D.; MILLER, A. Applying the theory of constraints principles and lean thinking at the marine corps maintenance center, Defense Acquisition Review Journal, 2004.

TELLES, M. A; PEREIRA, A. S; SEVERO, E. A; GUIMARÃES, S. C. F. Aplicação da Ferramenta IROG: Estudo de caso em uma empresa do ramo de autopeças em Caxias do Sul. Revista Global Manager, v. 14, n. 1, p. 74-92, 2014.

VEIT, D. R; GONCALVES, T; LACERDA, D. P; POSSEBON, A. P. Repercussões dos problemas conceituais do cálculo do IROG: Um estudo de caso sobre adoção de um software na indústria de transformados Termoplásticos. In: ENEGEP - Encontro Nacional de Engenharia de Produção, 32., 2011. Anais..., Belo Horizonte, MG, 2011.

WATSON, K. J.; PATTI, A. A comparison of JIT and TOC buffering philosophies on system performance with unplanned machine downtime, International Journal of Production Research, v. 46, n. 7, p. 1869-85. 2008.

YIN, R. Estudo de caso: planejamento e métodos. Porto Alegre: Bookman, 2005.

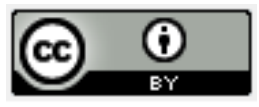

Artigo recebido em 12/08/2017 e aceito para publicação em 01/12/2017 DOI: http://dx.doi.org/10.14488/1676-1901.v18i3.2973 